\title{
III.
}

\section{Über Adenomyome und Pankreasgewebe in Magen und Dünndarm mit Beschreibung eines Falles von kongenitalem Duodenaladenomyom.}

\author{
Von \\ Elisabeth Weishaupt, \\ Leiterin des Pathologischen Instituts der Kgl. Universitäts-Frauenklinik Berlin (in Vertretung \\ von Prof. Robert Meyer). Direktor der Klinik Geh.-R. E. Bumm.
}

(Hierzu Taf. II.)

Die Veröffentlichung des vorliegenden Falles, bei dem es sich um eine erbsengroße Geschwulst des Duodenum unterhalb des Pylorus bei einem 11 Tage alten Kinde handelt, geschieht unter Zugrundelegung folgender Gesichtspunkte:

1. Gutartige Dünndarmtumoren, speziell Adenomyome, wurden bisher in spärlicher Zahl beschrieben; diejenigen in der Nähe des Pylorus nehmen eine Sonderstellung unter den Dünndarmtumoren ein und bilden Übergänge zu den Magentumoren, von denen sie nicht immer deutlich abzugrenzen sind.

2. Stehen die vorliegende und ihr ähnliche angeborene Neubildungen in ursächlichem Zusammenhange mit der kongenitalen Pylorusstenose?

3. Die Adenomyome des Verdauungstraktus bieten anatomisches und klinisches Interesse in bezug auf ihre Unterscheidung von Hyperplasien und von Divertikeln; dieselben Fälle werden von den Autoren verschieden gedeutet.

4. Das Vorkommen von Pylorus und von Dünndarmdrüsen einerseits und von Pankreaselementen andrerseits in der Pförtnergegend des Magens macht die eingehende Untersuchung der epithelialen Einschlïsse aller an der bezeichneten Stelle zu Gesicht kommenden Tumoren wünschenswert.

5. Adenomyome des Pylorus und des Dünndarms gelten zwar allgemein als angeboren; Beobachtungen an Föten und an Neugeborenen, die stets einen wichtigen Platz in dem Kapitel zum wenigsten der formalen Geschwulstgenese einnehmen werden, liegen aber bisher nur in geringer Zahl vor.

Die 32jährige Mutter des Kindes, Fünftgebärende, wurde wegen eklamptischer Anfälle (Schlaganfall!) in die Klinik eingeliefert, hatte im Wochenbett zahlreiche Anfälle und wurde 15 Tage post partum wegen Verdachts auf zerebrale Erkrankung nach der Charité-Nervenstation verlegt.

Kindersektion 138, 1914. 11 Tage gelebt. Kindesleiche von normaler Größe, weibl. Geschlechts; Hautfarbe gelblich. Gehirn ödematös, leicht ikterisch; kein Kernikterus. Lungen rosalackfarben; Herz o. B. Leber gelbbraun, Zeichnung deutlich, Milz klein. Zahlreiche Lymphknoten, die teilweise hämorrhagisch infiltriert sind, in der Radix mesenterii; Netz blutreich, Därme gebläht, Darmserosa injiziert. Auf der hinteren Fläche des Duodenum, unmittelbar unterhalb der Valvula pylori, ein reichlich erbsengroßer, sehr harter, verschieblicher Knoten, der nach außen in der Darmwand entwickelt ist. Darm, Magen durchgängig. BeiEröffnung des Darmes zeigen sich zahlreiche Defekte und blutig imbibierte Stellen in der Schleimhaut. 
Diagnose: Enteritis. Myoma duodeni. Die mikroskopische Untersuchung bestätigte die makroskopische Diagnose der Darmentzündung. Es lassen sich im Darmlumen und in der Sehleimhaut Gram-positive Kokken und Stäbchen in großer Zahl, auch Gram-negative Stäbchen, außerdem Epitheldefekte und entzündliche Infiltration des Schleimhantstroma nachweisen. Ein direkter ursächlicher Zusammenhang zwischen der infektiösen Darmerkrankung und dem Vorhandensein des Duodenaltumors kann natürlich nicht angenommen werden. Der kleine Tumor ist überdies nach der Außenseite des Darmes entwickelt und hat, wenn anch wahrscheinlich die Kontraktilität der Darmwand an der betreffenden Stelle beeinträchtigt, so doch keineswegs die Durchgängigkeit von Magen und Darm verhindert.

Der Tumor wurde in Seriensehnitte zerlegt, die $10 \mu$ dicken Schnitte meist nach van Gies on, einige mit Hämalaun-Eosin, Muzikarmin, Gram-Fuchsin und nach Best auf Glykogen gefärbt.

Mikroskopische Beschreibung: Auf dem transversalen Schnitt durch das Zentrum der Geschwulst und durch das Duodenum zeigt sich der Tumor aufgebaut aus epithelialen, bindegewebigen und muskulären Elementen, und zwar überwiegt das Epithel und das Muskelgewebe über das fibröse Gewebe. Einige mehr oder weniger scharf begrenzte Drüsenläppchen, die zum T'eil untereinander durch epitheliale, gewundene Schlïuche verbunden sind, werden von weitkalibrigen, vielfach ausgebuchteten Kanälen durchzogen, je 5-6 an der Zahl, die mit hochzylindrischem Epithel ausgekleidet sind (Fig. 1, Taf. II).

Die Zellen dieser weiten Kanäle stehen sehr dicht, so daß in den $10 \mu$ dicken Paraffinschnitten die Zellgrenzen nahe beieinander, die Zellen sogar teilweise übereinander gelagert sind. Das Protoplasma der Zellen erscheint nach van Gies on gefärbt von mittlerer Dunkelheit. Ein Kutikularsaum ist nur zum Teil, dann aber an mehreren nebeneinanderliegenden Zellen deutlich; vielfach ist der Innenrand der Zellen unseharf, vorgebuckelt, von Sekrettröpfchen überlagert. Die Zellkerne sind länglich, basal angeordnet, mit der Schmalseite der Zellbasis zugewendet. Zwischen den Zylinderzellen sind Becherzellen vorhanden, meist in großen Abständen, nur in wenigen Kanälen finden sie sich in beträchtlicher Zahl. Am Innenrande der Becherzellen fehlt der Kutikularsaum. Im Lumen der Kanäle lagert Sekret in feinen Tröpfchen, untermischt.mit Zellkernen. Eine Tunica propria ist nur stellenweise angedeutet. In die beschriebenen Kanäle münden geschlängelte Drüsengänge mit engem Lumen, deren Epithel aus sehr hellen Zylinderzellen mit schwach angedeutetem Kutikularsaum und flachem, quer gegen die Zellbasis gedrücktem Kern besteht. Die Zellen sind an der Basis breiter, gegen das Lumen schmaler, der Zelleib ist in der Mitte leicht gebuchtet. Die Tunica propria ist deutlicher als an den weiten Kanälen. Eingebettet sind die drüsigen Gebilde in ein lockeres, zytogenes Stroma, in dem Kapillaren und nach außen einzelne Muskelfasern, zuletzt einige fibrilläre Bindegewebszüge verlanfen. Der Mantel glatter Muskelfasern, der bis in die innere, die Ringmuskelschicht des Darmes zurückzuverfolgen ist, umgibt die Drüsenläppchen teilweise zirkulär, teilt sich aber auch in unregelmäßig breite Stränge, die in den Zwischenräumen zwischẹn den Lobuli wirr durcheinander verflochten sind. Zwischen den beschriebenen Drüsenläppchen und der Darmschleimhaút lagern, inmitten von glattem Muskelgewebe, Kanäle vom Typus der zuerst beschriebenen Gänge mit weiter, unregelmäßig geformter Lichtung, in die einige geschlängelte Drüsen mit engem Lumen einmünden. Der eine Gang erstreckt sich bis in die Submukosa hinein, von der sich die äußersten Lagen um Epithelgang, zytogenes Stroma und einige darin verlaufende glatte Muskelfasern als äußere, unscharfe Grenze herumschlagen. Die Längsmuskulatur des Darmes läßt sich in ihren äußersten Schichten auf der Außenseite der Neubildung verfolgen, während sich die inneren Züge mit den aus der Ringmuskelschicht stammenden verflechten. Die Ringmuskelschicht ist, verglichen mit Darmpräparaten von andern Neugeborenen, hypertrophisch. Die epithelialen Elemente der Geschwulst befinden sich in funktioneller Tätigkeit, wie aus den Sekrettröpfchen am Innenrande der Zellen, im Lumen der Kanäle, aus dem Vorhandensein der Becherzellen zu ersehen ist. Die Schleimfärbung fällt in den weiten Schläuchen stärker positiv aus als in den gewundenen Drüsen.

Die Darmschleimhaut auf demselben Schnitt zeigt sowohl Merkmale der Pylorus- wie der 
Dünndarmschleimhaut. Teils finger- teils keulenförmige Zotten sind auf 4 Schleimhautfalten aufgebaut, mit hochzylindrischen Epithelzellen bekleidet, die helles Protonlasma und kubische, basal angeordnete Kerne besitzen; einige Zellen haben eine Kutikula, der innere Teil der andern löst sich in amorphes Sekret auf, das auch die Räume zwischen den Zotten ausfüllt. Die beschriebenen Eigendrüsen besitzen meist in ihrem Grunde eine halsförmige Verengerung, unter der sich die Drüse in meist 2, seltener in 3 oder mehr Fortsätze gabelt. Auf einem kleinen 'Teil der Schleimhaut, etwa einem Fünftel, auf der dem Tumor gegenüberliegenden Darmwand, sind die Drüsen gewunden, ihre Lumina vielfach quer getroffen; die Muscularis mucosae durchbrechen sie an keiner Stelle, Die Schleimfärbung (Muzikarmin) ist nicht überall gleichmäßjig stark positiv, indessen sowohl in einem Teil der Eigendrüsen wie der Sekundürdrüsen deutlich. Die Bestsche Glykogenfärbung tritt in allen epithelialen Bestandteilen des Tumors und des Darmes sehr leuchtend rot, am stärksten in den gewundenen Drüsen der Neubildung hervor.

Im Verfolg von Serienschnitten nehmen die drüsigen Gebilde in den kleinen Läppchen der Geschwulst immer mehr zu; die Läppchen sind nach 60 Schnitten nicht mehr durch Muskelzüge, sondern nur durch einige Lagen fibrösen Gewebes voneinander getrennt und vereinigen sich nach 90 Schnitten zu einem Drüsengebilde, das mit andern Drüsenläppchen zusammenfließt und nach 260 Schnitten in offener Verbindung mit dem Darmlumen steht. Zahlreiche Quer- und Längsschnitte von gewundenen Kanälen mit Ausbuchtungen, Sprossen und einigen einmündenden Drüsen mit enger Lichtung liegen außer den größeren Drüsenbezirken in der Muskelgeschwulst, deren Faserbündel überall in unregelmäßigem Verlauf durcheinander verflochten sind. Die Darmschleimhaut weist dort, wo die Verbindnng mit den epithelialen Elementen der Neubildung besteht, zahlreiche, stark verzweigte Drüsen innerhalb der Submukosa auf (Brunnersche Duodenaldrüsen). Die weiten Epithelkanäle der Neubildung gehen von den Eigendrüsen des Darmes ab.

Auf den nä.chsten Schnitten erscheinen zwischen den Muskelzügen der Geschwulst in nächster Nïhe von den gewundenen Schleimdrüsen und in offener Verbindung mit diesen Drüsen epitheliale Schläuche mit niedrigen, unregelmäßig geformten Zellen, mit dunklem Protoplasma, meist dunkel tingierten, basalständigen Kernen, die, zu Läppehen angeordnet, den Pankreasdrüsen völlig gleichen. Der Übergang der Epithelarten und der Schlauchformen ist kein ganz schroffer, vollzieht sich aber auf einer nur wenige Zellen breiten Übergangszone, indem das Lumen der gewundenen Drüsen rasch enger wird, die Zellen niedriger, die Kerne größer und dunkler. Nach 70 Schnitten liegen 10 aus ca. 8-30 solcher Drüsenquerschnitte aufgebaute Läppchen zwischen den Darmdrüsen in der Submukosa (Fig. 2, Tafel II). Hier und da liegen, inmitten der Läppchen in kleinen Gruppen und Bändern um Gefäßkapillaren angeordnet, Zellen mit hellem Kern und hellem Protoplasma, die an die Zellen der Langerhanssehen Inseln erinnern. Einwandfrei und in ausgebildeter Form ließen sich indes diese intraazinösen Zellinseln nicht nachweisen. Die Darmmuskelschichten ziehen außen über die ortsungewöhnliche epitheliale Einlagerung hin; die Ringmuskelschicht ist dünner als auf der ibbrigen Darmperipherie, die Längsmuskelschicht dagegen nicht verändert. Die Darmschleimhaut ist über der Pankreasinsel gewulstet und in das Darmlumen hinein vorgestülpt, an anderer Stelle eingebuchtet, von Bindegewebs- und Muskelfasern unregelmäßig durchzogen. Die daraus entstehenden Drüsenläppchen enthalten Kanäle, die den anfangs beschriebenen weitkalibrigen Schläuchen der Neubildung entsprechen. Diese Kanäle stehen in Verbindung mit den Duodenaleigendrüsen, und wie sich aus diesen die Duodenaldrüsen (Brunnerschen Drüsen) entwickeln, so zweigen aus den Kanälen in den Drüsenläppchen der Geschwulst den Duodenaldrüsen völlig gleichende Drüsen ab.

Fasse ich die obige Beschreibung kurz zusammen, so ergibt sich bei einem 11 Tage alten Kinde das Vorhandensein einer Insel von Pankreasgewebe auf der dorsalen Seite des Duodenum innerhalb der Submukosa, unterhalb der Valvula pylorí. Die Pankreasschläuche und die gewundenen Duodenaldrüsen gehen an einigen wenigen Stellen unter rascher Annäherung des beiderseitigen Epithelund Schlauchcharakters offen ineinander über. Die über dem heteroplastischen Pankreas vorgebuchtete und daneben eingezogene Dünndarmschleimhaut ist vom Darminmen teilweise abge- 
schnürt und hat durch autonomes Wachstum ihrer distalen Elemente unter Beteiligung der Ringmuskulatur des Dünndarms zur Bildung eines erbsengroßen Adenomyoms geführt. Der Tumor ist nach außen vom Darm, subserös und unter den äußeren Fasern der Längsmuskelschicht entwickelt.

Die Darmringmuskulatur ist zirkulär um das Duodenallumen auf der Höhe der Gesehwulst hyperplastisch.

Pylorusdrüsen, die sich nicht durch Struktur oder mikrochemisches Verhalten, sondern nur durch ihre Beschränkung auf das Stratum proprium der Mukosa von den Duodenaldrüsen unterscheiden lassen, greifen auf den Dünndarm über.

Das Vorhandensein des heteroplastischen Pankreas und des Adenomyoms stehen in ursächlichem Zusammenhang miteinander; der T'umor ist indessen nicht von dem Pankreasgewebe selbst, sondern von der Schleimhaut des Duodenum ausgegangen, deren abgeschnürte, 'selbständig gewordene Elemente ihrerseits wiederum wahrscheinlich einen formativen Reiz auf das Muskelgewebe ausgeübt haben.

Der Tumor enthält alle Gewebe seines Mutterbodens, der Darmschleimhaut und der Ringmuskulatur und besitzt demzufolge einen organoiden Bau.

Durch die Wandverdickung mehr als durch den nach außen entwickelten Tumor wurde unterhalb der Valvula pylori eine Stenose des Dünndarms verursacht, die indessen nicht zum Verschluß des Lumens führte.

Nunmehr gehe ich auf die anfangs bezeichneten Gesichtspunkte ein, die zur Veröffentlichung der beschriebenen Neubildung führten.

In unmittelbarer Nähe des Pylorus gelegen, charakterisiert sich die Geschwulst als ein echter Dünndarmtumor, dessen epitheliale Bestandteile aus der Schleimhaut, das Muskelgewebe aus der Ringmuskulatur des Darmes hervorgegangen sind, nicht wie in einigen Fällen anderer Autoren aus der Muscularis mucosae. Auch der Ursprung aus der Gefäßmuskulatur ist abzulehnen. Die Gewebe des Magens haben keinen Anteil an dem Aufbau der Geschwulst, dagegen finden sich Pylorusdrüsen auf der Höhe des Tumors in der Darmdrüsenschleimhaut, was an dieser Stelle als physiologisch zu bezeichnen ist.

Wie der vorliegende Fall zeigt, können selbst kleine, nach außen entwickelte, in der Nähe des Pylorus gelegene Geschwülste mittelbar eine Stenose des Intestinaltraktus veranlassen, die hier durch teilweise Vorbuchtung der Schleimhaut und durch die zirkuläre Hypertrophie der Darmmuskulatur zustande gekommen ist.

Eine Verwechslung zwischen Geschwulst und Hyperplasie der Darmmuskulatur glaube ich ablehnen zu können, da Epithel und Muskelgewebe ein selbständiges, regelloses Wachstum zeigen. Der Befund von heterogenem Pankreasgewebe ist nicht selten, die Fähigkeit der Schleimhaut von Magen und Dünndarm, Pankreasgewebe zu entwickeln, bekannt. Nicht vorkommen tut dasselbe in Ösophagus und Dickdarm. Die Frage ist, ob die ortsungehörige Bildung als Atavismus, als Dysplasie oder als Prosoplasie aufzufassen ist. Bei manchen Tieren, z. B. bei Pelobates fuscus (zu den Anuren gehörig), beim Erdsalamander und beim Maulwurf ist die 
Teilung des Pankreas in mehrere Drüsen und die innige Verschmelzung mit der Darmwand das Normale ${ }^{1}$ ).

Ich habe noch mehrfach Pankreasinseln im Dünndarm von Neugeborenen gefunden, doch nur in dem vorliegenden Falle lagen dieselben in der dorsalen Wand des Duodenum und in der Submukosa, während sie in den übrigen Fällen in der ventralen Wand, in der Gegend des Pankreaskopfes, subserös entwickelt waren.

Die verschieden gelagerten Pankreasinseln müssen meiner Ansicht nach in bezug auf ihre Entstehung verschieden gedeutet werden. Diejenigen, die sich in der Darmwand dort finden, wo normaler- oder abnormerweise Ausführungsgänge der Bauchspeicheldrüse angelegt werden, oder dort, wo sich mit oder ohne Ausführungsgang ein akzessorisches Pankreas außerhalb des Darmes diesem anlegt, werden durch eine frühzeitige Aussprossung des Ausführungsganges, durch ein Verschmelzen von Darmwand und Pankreasanlage, also durch eine illegale Gewebsverbindung zustande gekommen sein. Diejenigen Pankreasinseln dagegen, die innerhalb der Darmwand, mitten in der Schleimhaut, an einem für den Menschen nicht normalen, in der Stammesgeschichte aber als Entstehungsort gekennzeichneten Platze liegen, sind m. E. phylogenetisch als lokaler Rückschlag, ontogenetisch als Prosoplasie des Darmepithels aufzufassen.

Im folgenden stelle ich zusammen, was aus der Literatur als Erklärung und Erweiterung des Vorhergehenden von Interesse sein dürfte.

Kaufmann ${ }^{2}$ ) sagt, daß die angeborene Stenose des Pylorus meist mit starker, muskulärer Hypertrophie verbunden sei, ihre Ätiologie aber noch strittig wäre. Nach Kaufmann gibt es echte angeborene Stenosen mit kompensatorischer Hypertrophie, während in andern Fällen eine spastische Kontraktur mit nachfolgender Arbeitshypertrophie des Pylorus vorhanden sein soll. Manche Autoren nehmen auch einen Spasmus des gesamten Magens an oder eine Funktionsstörung der Nerven, welche die koordinierte Muskelbewegung von Magen und Pylorus besorgen, was einen Antagonismus und schon in utero, bei Aufnahme des Fruchtwassers, eine fortwährende Arbeit und so eine funktionelle Hyperplasie der Muskulatur veranlasse. Andere machen eine geschwulstartige Hypertrophie der Ringmuskulatur mit gleichzeitiger Bindegewebsvermehrung oder eine wulstige Verdickung der Mukosa für die angeborene Pylorusstenose verantwortlich.

Des weiteren macht Kaufmann darauf aufmerksam, daß um versprengte Pankreasläppchen kleine Adenomyome entstehen können.

Die erste Angabe in der deutschen Literatur, die ich über einen adenomatösen Tumor des Dünndarms fand, stammt von Wagner ${ }^{3}$ ). Derselbe berichtet über

1) Engel, Österr. med. Jhrb. 33. Bd. - Zenker, Virch. Arch. 21. Bd. - Friedrich, ZiemBens Hdb. d. spez. Path. u. Ther. 8. Bd. - Haane, Arch. f. Anat. u. Phys., Anat. Abt. 1905. - Derselbe, Arch. f. wiss. u. prakt. Tierheilk. 31. Bd., 1905. - Keibe1 und Mall, Hdb. d. Entw. - Baldwin, Anat. Record. 5. Bd., 1911.

2) Kaufmann, E., Lehrb. d. spez. path. Anat., 5. Aufl., 1909.

3) Wagner, E., Vortrag in der mikrosk. Ges. zu Leipzig, 1859. 
einen kleinen, gestielten Tumor am Ende des Ileum, dessen epitheliale Struktur im großen und ganzen jene der Lieberkühnschen Drüsen wiederholte.

Weiter beschreibt Salvioli') ein Adenom im Duodenum, Langhans ${ }^{2}$ ) einen ungestielten Drüsenpolypen im Ileum.

In dem einen von Carbones ${ }^{3}$ ) 3 Fällen von Adenomen im Duodenum ähnelten die Drüsenschläuche den Lieberkühnschen Drüsen, in den beiden andern vermutet $\mathrm{Carbone}^{3}$ ) ihren Ursprung in akzessorischen Pankreasanlagen.

Gegenüber dem Mesenterialansatz im Dünndarm eines nicht ausgetragenen Neugeborenen fand Durante ${ }^{4}$ ) einen erbsengroßen, gut abgegrenzten, ungestielten Tumor, der in der Mitte eine mit Zylinderepithel ausgekleidete Höhle mit Seitenkanälen enthielt. Muskelfasern umgeben die Drüsengänge, die Fasern laufen parallel mit denen des Darmtransversalschnittes. Durante ist unsicher, ob es sich um ein wirkliches Adenomyom oder um ein atrophisches Darmdivertikel handelt.

Lubarsch ${ }^{5}$ ) beschreibt ein reichlich kirschgroßes Adenomyom in der Pförtnergegend des Magens. „Es handelt sich“, wie Lubarsch sagt, ,um ein aberriertes Pankreas, das submukös gelegen ist, dessen mit hohem Zylinderepithel ausgekleidete Ausführungsgänge in erheblicher Weise proliferiert waren und auch ihrerseits wiederum eine Wucherung der sie umgebenden Muskulatur bewirkten."

Bei den 4 ersten, von Erwachsenen stammenden Fällen von Magnus-Alsleben ${ }^{6}$ ) ist ein direkter Zusammenhang zwischen den Brunnerschen Drüsen der Dünndarmschleimhaut und den genau ebenso gebauten Drüsen der Tumoren vorhanden. Der 5. Fall betrifft ein 10 Monate altes Kind, bei dem die Drüsen des Tumors ihren Ausgang von den Pylorusdrüsen genommen hatten. MagnusAlsleben bezeichnet alle 5 Fälle als richtige Adenomyome.

Torkel ${ }^{7}$ ) beschreibt eine diffuse Muskelhyperplasie am Pylorus von einem 4. Wochen alten Kinde, die er für gleichartig mit den 5 Fällen von Magnus-Als leben hält; er lehnt eine Einreihung dieser und seines eigenen Falles unter die Geschwülste, die Adenomyome oder Adenome, ab.

Über einen Mischtumor, ein Fibromyolipozystadenom, an dem Dünndarm eines 6jährigen Knaben berichtet Gallus Bauer ${ }^{8}$ ).

1) Salvioli, Contribuzione allo studio degli adenomi. Osservatore, Gazzetta delle cliniche di Torino. Torino 1876.

$\left.{ }^{2}\right)$ Langhans, Th., Über einen Drüsenpolyp im lleum. Arch. f. path. Anat. Bd. 38, H. 4.

3) Carbone, Über Adenomgewebe im Dünndarm. Zieglers Beitr. Bd. 5, 1889.

4) Durante, Adéno-myome congénital de l'intestin usw. Bull. d. 1. soc. anat. d. Paris, 1901.

5) Lubarsch, Hyperplasie und Geschwülste. Ergebn. d. allg. Path., Morphol. u. Phys. Abt. II, Bd. 4, S. 330, 1895 und Jahrg. II, 1897, S. 570.

$\left.{ }^{6}\right)$ Magnus-Alsleben, E., Adenomyome des Pylorus. Virch. Arch. Bd.173, S. 137--155, 1903.

$\left.{ }^{7}\right)$ Torkel, Die sog. kongentiale Pylorushyperplasie eine Entwicklungsstörung. Virch. Arch. Bd. 180, 1905.

8) Gallus Bauer, Inaug.-Diss. Würzburg, 1906. 
Versé ${ }^{1}$ ) hält ein Myoadenom des Dünndarms bei einem 57jährigen Manne für eine kongenitale Bildung. Ein zweiter Fall betrifft ein Fibromyoadenom mit spärlichen epithelialen Bildungen bei einem 48jährigen Manne, ein dritter $2 \mathrm{~m}$ oberhalb der Ileozökalklappe wird von Versé als Rest des Ductus ompahlomesentericus gedeutet.

Von den 3 Trappeschen ${ }^{2}$ ) Fällen von Adenomyomen des Magendarmtraktus fanden sich 2 im Dünndarm, das erste, am unteren Ileum eines 54jährigen Mannes, war vollständig in der Submukosa entwickelt, die Muskulatur stammte von der Muscularis mucosae. Das zweite, gleichfalls am unteren Ileum, wies Drüsenläppchen vom azinösen Bau der Speicheldrüsen auf und leitet nach Trappes Meinung zu den von Thorel und von Carbone beschriebenen Fällen von Nebenpankreas iiber. Trotzdem die Entwicklung der Gefäße in den Tumoren auffallend stark ist, weist Trappe die Genese des Muskelgewebes der Darmadenomyome von der Muskulatur der Gefäße ab, wozu Cohen ${ }^{3}$ ) dagegen eventuell geneigt ist. Cohen stellt 28 Muskelgeschwülste vom Magendarmtraktus zusammen, die aber nicht alle epitheliale Einschlüsse besaßen. In einem kirschgroßen Tumor bei einer 50jährigen Frau stimmten dieselben sicher mit Pankreasgewebe überein.

Thorel ${ }^{4}$ ) beobachtete Entwicklungsanomalien des Pankreas bei 7 männlichen Individuen zwischen dem 21. und 53. Lebensjahre. Zwei der Fälle können seiner Meinung nach als Adenomyome gelten.

Vielfach finden sich Inseln von Pankreasgewebe im Dünndarm in Verbindung mit Divertikeln, so daß die Möglichkeit eines ursächlichen Zusammenhangs zwischen beiden Bildungen erörtert wird. Ein Pancreas accessorium oder aberrans liegt zuweilen an der Spitze oder auch im Mesenterium eines Meckelschen Divertikels. Das Pancreas accessorium kann auch selbst zu Divertikelbildung führen ${ }^{5}$ ). Jedenfalls kommen Divertikel und Pankreasinseln sowohl zusammen wie jedes für sich zur Beobachtung.

Trappe (a. a. O.), Albrech $\mathbf{t}^{6}$ ) und andere Autoren ${ }^{6}$ ) vertreten die Anschauung,

1) Versé, Über Entstehüng, den Bau und das Wachstum der Polypen, Adenome und Karzinome des Magen-Darmkanals. Path. Inst. d. Univ. Lejpzig, H. 5, Bd. 1, S. 1-175, 1908.

2) Trappe, Uber geschwulstartige Fehlbildungen von Niere, Milz, Haut und Darm. Frankf. Ztschr. f. Path. Bd. 1, S. 109-123, 1907.

$\left.{ }^{3}\right)$ Cohen, Beitr. z. Histologie u. Histogenese der Myome des Uterus und des Magens. Arch. f. path. Anat. u. Phys. Bd. 158, H. 3, 1899.

4) 'T'horel, Histologisches über Nebenpankreas. Arch. f. path. Anat. u. Phys. Bd. 173, H. 2, S. 281-302, 1903.

5) Kaufmann, Lehrb. d. spez. path. Anat. S. 620, 1909. - Meyer, Robert, Zur Kenntnis der normalen und abnormen embryonalen Gewebseinschlüsse und ihre pathologische Bedeutung. Ztschr. f. Geb. u. Gyn. S. 221-320, 1912 (Literatur). - Derselbe, Erg. d. Path. Bd. 9 u. 15. - Linsmayer, Bericht über 46 Duodenaldivertikel. 17. Tagung der D. Path. Ges. in München vom 23.-25. März 1914. Ztlbl. f. allg. Path. u. path. Anat. Bd. 25. Helly, Orth, Simmonds, Diskussionsbemerkungen zu obigem Bericht.

6) Albrecht und Arzt, Frankf. Ztschr. f. Path. Bd. 4, 1910. - Albrecht, Erg. d. Path. Bd. 15, S. 878, 1912. — Thilemann, Deutsche Ztschr. f. Chir. Bd. 85, 1906 (Literatur). - 
daß Pankreasgewebe in der Darmwand auf weitere Differenzierung iiberschüssig gebildeter Darmdrüsenepithelien zurïckzuführen ist, denen ,ebenso wie der ganzen Darmanlage überhaupt, die Fähigkeit zur Pankreassbildung zuzuschreiben ist". Ob Trappe und Albrecht dem Darmepithel bzw. der Darmanlage die Fähigkeit zu dieser Prosoplasie während der ganzen Lebensdauer oder nur während des fötalen Lebens zuschreiben, geht aus ihren Ausführungen nicht hervor.

Im allgemeinen werden die ortsungewohnten Gewebe, so auch das heteroplastische Pankreas, gleichzeitig mit, dem ortsgewöhnlichen Gewebe angelegt, und die Entwicklung der ortsungewohnten Gewebe dürfte in der Regel ebenso zeitlich begrenzt sein, wie es ortlich ist.

Ribbert, Cohen und Thorel sind der Meinung, daß es sich in den Fällen, wo bei Epitheleinschlüssen der Darmwand oder in Adenomyomen der Charakter von Pankreasgewebe nicht deutlich ist, wo aber am Ende der epithelialen Schläuche kleine Drüsenläppchen vom Bau der Speicheldrüsen vorhanden sind, um eine Rückbildung oder um mangelhafte Entwicklung von aberrierten Pankreaskeimen handelt.

Die primären Karzinome des Processus vermiformis, die nach den bisherigen Erfahrungen einen mit dem sonstigen Charakter der malignen epithelialen Geschwülste nicht übereinstimmenden Mangel an Wucherungs- und Metastasierungsneigung bekunden, die deshalb als Karzinoide bezeichnet werden, sollen möglicherweise von aberriertem Pankreasgewebe abstammen. R. Meyer (a.a. O.), der eine ausführliche, übersichtlich angeordnete Zusammenstellung aller bisher veröffentlichten Befunde von normalen und abnormen embryonalen Gewebseinschlüssen gibt, betont, daß eine den karzinoiden Knötchen im Proc. vermiformis entsprechende kongenitale Grundlage bisher nicht nachgewiesen sei (Lit. über Appendixkarzinoide s. unten) ${ }^{\mathbf{1}}$ ). Daß eine zunächst als primäres Karzinom oder Karzinoid des Proe. vermiformis angesprochene Neubildung sich später als Metastase oder Rezidiv entpuppen kann, liegt auf der Hand, und wurde an einer eigenen Beobachtung ('T. 1364) erfahren, bei der der Uterus 5 Monate vorher wegen Portiokarzinom exstirpiert war.

Robert Meyer (a. a. O.) stellt folgende Beobachtungen normaler und heteroplastischer Pankreasanlagen bei Föten und Neugeborenen zusammen.

Simon, Diss. Erlangen, 1907 (Literatur). - Oppel, Lehrb. d. vergl. mikr. Anat. Bd. 3. -

Nazari, Boll. Acad. Roma, 1909, Bd. 20. - Lécène, Soc. anat. d. Paris, fevr. 1912. Hulst, Ztlbl. f. Path. Bd. 20, 1909.

$\left.{ }^{1}\right)$ Heinrich, Virch. Arch. Bd. 98, 1909. - Reitmann, Path. d. Bauchspeicheldrüse. Ztschr. f. Heilk. H. 26, 1905; H. 1 u. 27, 1906; H. 4. - Markwald, Münch. med. Wschr. 1905. Oberndorfer, Frankf. Ztschr. f. Path. Bd. 1, 1907. - Versé, Arb. a. d. path. Inst. Leipzig. 1908. - Goetjes, Frankf. Ztschr. f. Path. Bd. 9, 1910. - Milner, D. med. Wschr. 1910. Mandl, Ztschr. f. Gyn. 1911. - Simmonds, Münch. med. Wsehr. S. 501, 1912. - Lahm, Ztschr. f. Geb. u. Gyn. Bd. 77, 1915. - Kudo, Ztschr. f. Krebsforschung 1908. v. Gaza, Münch. med. Wschr. Nr, 14 u. 25, 1910. - Miloslavich und Namba, Ztschr. f. Krebsforschung Bd. 12, 1912. - Rogg, Ztschr. f. Krebsforschung Bd. 13, 1913. 
Bei einem Fötus von $20 \mathrm{~mm}$ sah er einen kurzen Schlauch in der ventralen Duodenalwand, den er für die erste Anlage des Pankreas hält.

Weiter fand Robert Meyer in der Ventralwand des Duodenum bei einem Fötus von 28 mm Länge ein Pankreas, außerdem bei demselben Fötus einen Iangen Kanal in der Wand des Pylorus mit ventraler Mündung. Der Kanal, der in der Submukosa verläuft und sich in zwei Enden spaltet, wird vom Autor als ein akzessorisches, heteroplastisches Pankreas an ungewöhnlicher Stelle gedeutet.

Von einem Neugeborenen mit Hernia diaphragmatica beschreibt Robert Meyer den sehr ungewöhnlichen Befund einer Pankreasverlagerung in die Magenwand bis in den Ösophagus hinauf. In diesem Falle mündeten die Ausführungsgänge an richtiger Stelle in das Duodenum.

Auch R. Meyer fand nicht selten Pankreasgewebe an Darmdivertikeln, wie überhaupt Drüsenanlagen an der Spitze von Divertikeln allgemein in der Entwicklung za beobachten seien. Die Ansicht, daß das Divertikel durch Zug sekundär entstehen könnte, wie andere Autoren, u. a. Kaufmann, annehmen, ist ihm nicht verständlich.

Ganz kurz möchte ich noch die Anschauung einiger Autoren über die Identität der Pylorus- und Duodenaldrüsen und über physiologische und abnorme Unregelmäßigkeiten des Epithels in Magen und Darm referieren.

Daß in bezug auf die Schleimhaut zwischen Magen und Dünndarm eine scharfe Grenze nicht gezogen werden kann, wurde bereits oben erwähnt. Duodenaldrüsen reichen in den Magen hinein, und Pylorusdrüsen erstrecken sich auf den Anfangsteil des Duodenum. Aber auch in größerer Entfernung vom Pylorus findet sich im Magen typisches Darmepithel und sind, in einem Falle (Poindecker ${ }^{1}$ )), Magenschleimhautinseln im Dünndarm beschrieben worden.

Die letztgenannte Beobachtung steht im Gegensatze zu Schriddes ${ }^{2}$ ) Theorie über ortsunterwertige und über ortsdominierende Schleimhautelemente im Magendarmkanal, nach der die ortsunterwertigen nur kranial von denjenigen Abschnitten vorkommen, in denen dieselben ortsdominierend sind.

Auch die Befunde von Magenschleimhaut in Meckelschen Divertikeln, in Nabelzysten und Nabeladenomen dürften nicht leicht mit der Schriddeschen Theorie in Einklang zu bringen sein.

Beschreibungen von Darmepithel in der Magenwand liegen vor von Jouvenel ${ }^{3}$ ), Hari ${ }^{4}$ ), Schmidt ${ }^{5}$ ), Lubarsch ${ }^{6}$ ), Schaffer ${ }^{7}$ ), v. Ebner ${ }^{8}$ ).

1) Poindecker, H., Über einen Fall heterotoper Magenschleimhaut im Dünndarm. Ztlb]. f. allg. Path. u. path. Anat. Bd. 23, Nr. 11, S. 481, 1912.

2) Schridde, Die ortsfremden Epithelgewebe d. Menschen. Samml. anat. u. phys. Aufs. Gaupp u. Nagel, Jená 1909.

$\left.{ }^{3}\right)$ Jouvenel, Journ. de l'an. et de la phys., 42. Jahrg., 1906.

4) Hari, P., Arch. f. mikr. Anat. u. Entw., 58. Bd., S. 685-726, 1901.

5) Schmidt, Virch. Arch. f. path. Anat. u. Phys. 143. Bd., S. 483.

-) Lubarsch, Achilia gastriea mit einem anat. Beitr., S. 32. O. Lubarsch, 1897.

6) Lubarsch, Unregelm. d. Fpithelgrenzen in Magen, Darm, Gallenblase. D. Path. Ges., 1906. 
Zwischen Pylorus- und Intestinaldrüsen besteht zum mindesten eine enge funktionelle und morphologische Verwandtschaft.

Deimler ${ }^{1}$ ) spricht sich für die Identität der Pylorus- und der Duodenaldrüsen aus. Gegen diese Annahme spricht nach ihm kein einziger Grund, dafür folgende Punkte: Der ganz langsame Übergang der einen Drüsenart in die andere, das Vorkommen von Anastomosen beider Drüsenarten, ihr gleiches Verhalten bei künstlich hervorgerufenen Funktionszuständen, z. B. nach Pilikarpin-Injektionen. Beide Drüsenzellen verhaiten sich sowohl auf die feinsten Sekretgänge als auch tinktoriell und chemisch ganz gleich.

Im Gegensatze zu Deimler äußern sich Scheunert und Grimmer ${ }^{2}$ ) dahin, daß nach ihren Versuchen beim Pferd, Schwein und Rind erhebliche Unterschiede in den Funktionen beider Drüsen vorhanden sind. Wenn die fraglichen Drüsen funktionell nicht identisch sind, so können sie es nach Scheunert und Grimmer auch morphologisch nicht sein, sondern es müssen histologische Unterschiede vorliegen, wenn solche auch mit den jetzigen Hilfsmitteln nicht nachweisbar sind.

Zum Schlusse meiner Ausführungen möchte ich noch auf die Möglichkeit eines Zusammenhanges von heteroplastischem Pankreas in der Pylorusgegend mit malignen Neubildungen an dieser Stelle hinweisen, ein Zusammenhang, ähnlich wie ihn, was bereits oben gesagt wurde, einige Autoren für die Karzinoide des Proc. vermiformis mit daselbst kongenital verlagertem Pankreas hypothetisch angenommen haben. Für die Pylorusgegend erscheint die Annahme um so viel näher liegend, als hier sowohl Pankreasinseln wie primäre maligne Epithelgeschwülste relativ häufig beobachtet werden, während am Wurmfortsatz die primären bösartigen Neubildungen überaus selten und Pankreasgewebe dort bisher überhaupt niemals gefunden wurde. Es könnten Karzinome, die lokale und individuelle Wachstumsdisposition vorausgesetzt, sowohl direkt aus den Zellen der Pankreasinsel hervorgehen oder auch indirekt aus Wucherungen der umgebenden Magenoder Darmschleimhaut, wie der beschriebene Fall ein Paradigma für die Förderung der Entstehung einer gutartigen Geschwulst durch das ortsungewöhnliche Gewebe $\mathrm{zu}$ sein scheint.

\section{Erklärung der Figuren auf Taf. II.}

Fig. 1. 'l'ransversalschnitt durch Adenomyom und durch Dünndarm. Lupenvergrößerung. Färbung nach van Gieson. Weite Epithelschläuche mit Becherzellen, gewundene Drüsen, die in die weiten Kanäle einmünden.

Fig. 2. Obj. 3, Okul. 1, Tub. 175. Färbung nach van Gieson. $P$ Pankreasgewebe, $D r d r$ Darmdrüsen. Pankreasinsel in der Submukosa des Dünndarms.

7) Schaffer, Sitzungsber. d. Kais. Akad. d. Wiss. in Wien, Abt. III, 1897, Okt.

${ }^{3)}$ v. Ebner, Köllikers Handb., 6. Aufl., Bd. 4, 1899.

1) Deimler, Über die Pylornsdrüsenzone des Magens usw. Intern. M. f. Anat. u. Phys. Bd. 22, 1905.

2) Scheunert und Grimmer, Intern. M. f. Anat. u. Phys., Bd. 23, 1906.

Virchows Archiv f. pathol. Anat. Bd. 223. Heft 1. 


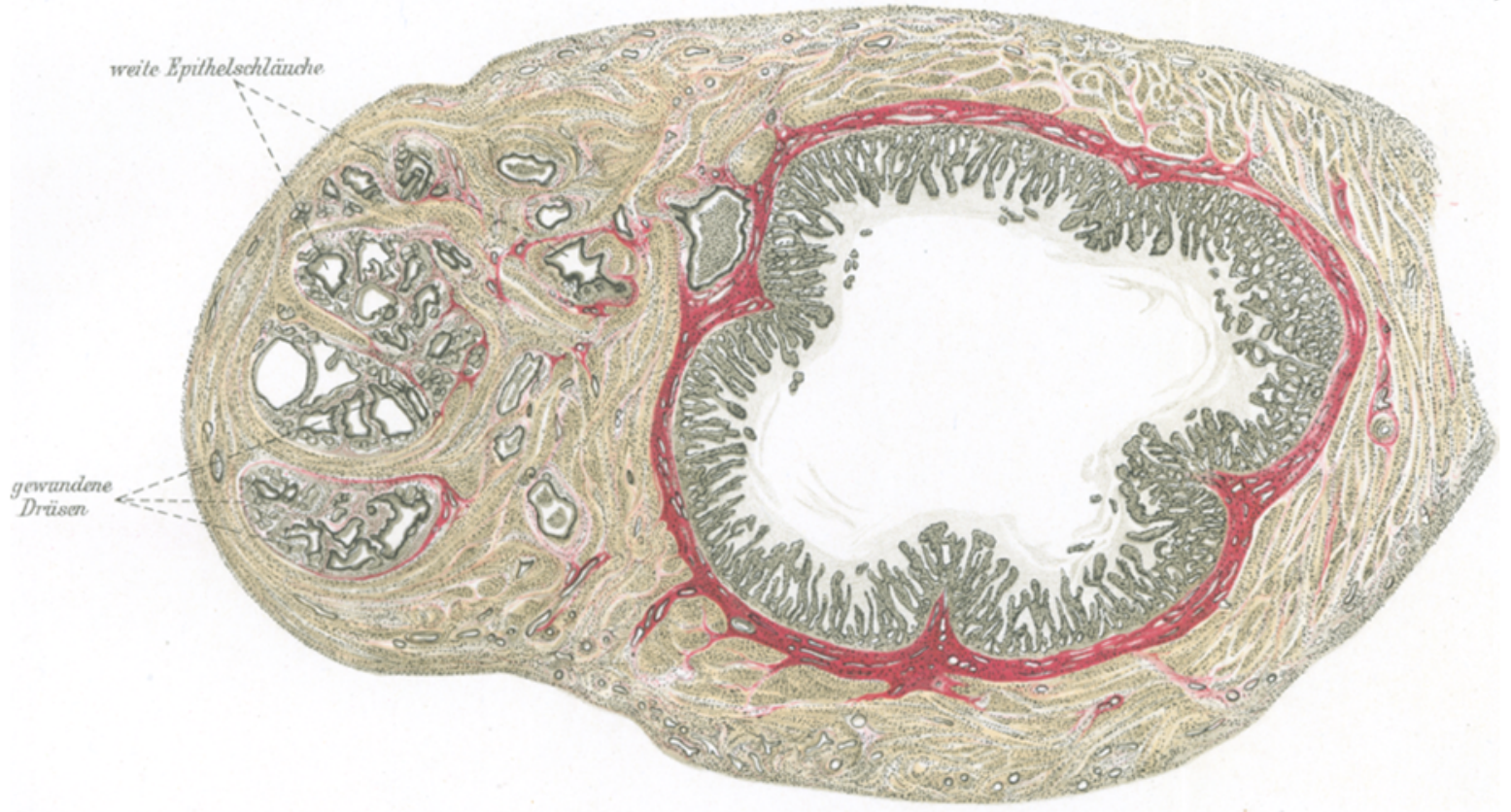

Fig.1.

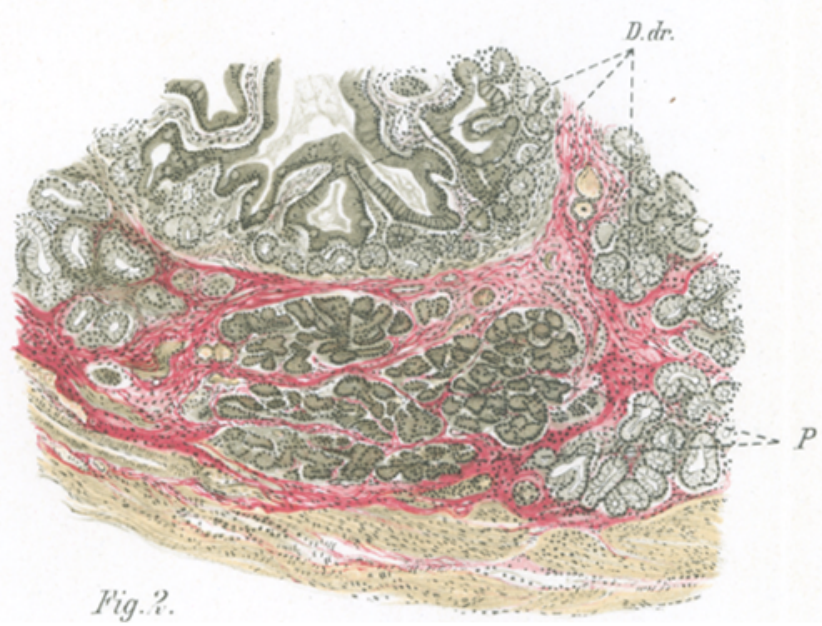

